\title{
Quantum Confinement Effect on Photoluminescence of Nanocrystalline ZnO Thin Films
}

\author{
Manju Arora, Santosh Singh and Sukhvir Singh \\ CSIR-National Physical Laboratory, Dr. K.S. Krishnan Road, New Delhi, India
}

\begin{abstract}
Nanocrystalline $\mathrm{ZnO}$ thin films with different morphology were grown on silicon substrate by sol-gel process using different zinc acetate sol concentrations. These films were characterized by high resolution XRD, SEM, EDS, photoluminescence (PL) and UV-VIS spectroscopic techniques to reveal their structural, morphological and optical details. The blueshift in strong free excitons recombination peak is observed as the morphology of the film changes from spindle shape to nanorods to clustering of nanorods with increase in sol concentration due to quantum confinement effect in the nc-ZnO thin films.
\end{abstract}

Keywords: Quantum confinement, Photoluminescence, Nanocrystalline, $\mathrm{ZnO}$, Thin films

\section{INTRODUCTION}

Nanocrystalline (nc)-ZnO has drawn considerable attention in the fields of optics, optoelectronics, sensors, and actuators due to its semiconducting, piezoelectric, and pyroelectric properties [1-2]. ZnO nanostructures exhibit high specific surface area, nontoxicity, chemical stability, electrochemical activity and high electron communication features. The origin of PL band of $\mathrm{ZnO}$ is still not fully understood. Some authors attributed the PL band to the recombination of excitons [3-4] while the others think that the emission is from surface impurities or defects [5-6], Hence the identification of PL peaks becomes very important in these investigations.

In understanding, the quantum confinement effect on PL spectra of $\mathrm{ZnO}$ thin films with different morphologies, the size, geometry and the concentration of impurities and defects are considered. In exploring this effect, it is found that the size of particles should comparable with the Bohr radius of $\mathrm{ZnO}$ with narrow size distribution. The geometry of particles and the concentration of impurities and defects prime parameters should be very low so that the PL signal of impurities or defects should not dominate in the PL spectra.

\section{EXPERIMENTAL DETAILS}

Nanocrystalline $\mathrm{ZnO}$ thin films on silicon $(\mathrm{Si})$ substrate by sol gel method. Before deposition the surface of Si substrate was oxidized by boiling the wafer in $70 \% \mathrm{HNO}_{3}$ and forms $\mathrm{SiOH}$ which improves its adhesion with deposited film. Zinc acetate $\left[\mathrm{Zn}\left(\mathrm{CH}_{3} \mathrm{COO}\right)_{2} \cdot 2 \mathrm{H}_{2} \mathrm{O}\right]$ was used as precursor for sol preparation and turbidity of sol solution was removed by adding diethanolamine. Si wafer with drop of sol was allowed to spin at the rate of $3000-4000 \mathrm{rpm}$. Followed by drying the coated wafer at $100^{\circ} \mathrm{C}$ and subsequent annealing at $350{ }^{\circ} \mathrm{C}$ for one hour. Multiple coatings were done to obtain the workable thickness of the film. The ellipsometric data show that for ten coatings, the film thickness was only of the order of 2000-2500 $\AA .10 \%, 15 \%$ and $25 \%$ of zinc acetate sol concentrations were used for $\mathrm{ZnO}$ film deposition. These $\mathrm{ZnO}$ films were characterized by Bruker AXS D8 Advance diffractometer which has in built Diffrac ${ }^{\text {plus }}$ software, using CuK $\alpha$ radiation for structural details, LEO -440 SEM with EDS attachment, Perkin Elemer Photoluminescence and Shimazu UV-VIS spectrometers were for morphological, structural and optical characterization of these films.

\section{RESULTS \& DISCUSSIONS}

The formation of zinc oxide by sol-gel process using zinc acetate as reaction precursor can be discussed by the following chemical reaction mechanism. The formation of zinc oxide by sol-gel process proceeds via hydrolysis, condensation and poly-condensation

16th International Workshop on Physics of Semiconductor Devices, edited by Y. N. Mohapatra, B. Mazhari, M. Katiyar, Proc. of SPIE Vol. 8549, 85492D · (c) 2012 SPIE · CCC code: 0277-786/12/\$18 · doi: 10.1117/12.927415 
reactions. As we know that zinc acetate on dissolving in water is partially hydrolyzed and the rest is ionized. The extent of hydrolysis depends upon the availability of water from the ambient atmospheric humidity. The hydrolysis of zinc acetate leads to the formation of the basic zinc acetate, which on evaporation of the water, does not give pure zinc acetate but produces a mixture of zinc acetate and basic zinc acetate. On heating, ionized zinc acetate form zinc hydroxide and acetic acid which evaporates off while basic zinc acetate hydrolyses to zinc hydroxide and acetate ions. After the evaporation of the water molecules, this would result in a final product which can be written as $\mathrm{HO}-\left(\mathrm{Zn}-\mathrm{O}-\mathrm{Zn}-_{n} \mathrm{OH}\right.$ where $n$. is the number of molecules taking part in the condensation process (poly-condensation). Since during the course of poly-condensation, the reaction proceeds uniformly in all directions in the plane of the substrate, the process of crystallization becomes steady and thus uniform.

Zinc oxide crystallizes in the form of a wurtzite structure with alternating planes composed of tetrahedrally coordinated $\mathrm{Zn}^{2+}$ and $\mathrm{O}^{2}$ - stacked alternately along c-axis of hexagonal unit cell with $a_{0}=0.3250 \mathrm{~nm}$ and $\mathrm{c}_{0}=0.5207 \mathrm{~nm}$ as shown in Fig. 1. XRD pattern of sol gel deposited nanocrystalline $\mathrm{ZnO}$ thin film is presented in Fig. 2. shows diffraction peaks at $2 \theta$ (in degrees) 31.680 , $34.340,36.180,56.540,62.780$ and 68.260 of [100], [002], [101], [110], [103] and [112] planes respectively. This confirms the polycrystalline nature of the deposited film with some preferential orientation of [101] plane. The calculated parameters of unit cell are $\mathrm{a}=0.3255(0.0004) \mathrm{nm}$ and $\mathrm{c}=0.5216(0.0008) \mathrm{nm}$. These are very close to the reported PDF\#36-1451 data $\left(\mathrm{a}_{0}=0.3250\right.$ $\mathrm{nm}$ and $\mathrm{c}_{0}=0.5207 \mathrm{~nm}$ ) The crystallite size of $\mathrm{ZnO}$ thin film was measured by using Sherrer formula. In these $10 \%, 15 \%$ and $25 \%$ sol concentration grown $\mathrm{ZnO}$ thin films have particle size calculated from [002] peak in the range of 20 to $30 \mathrm{~nm}$.

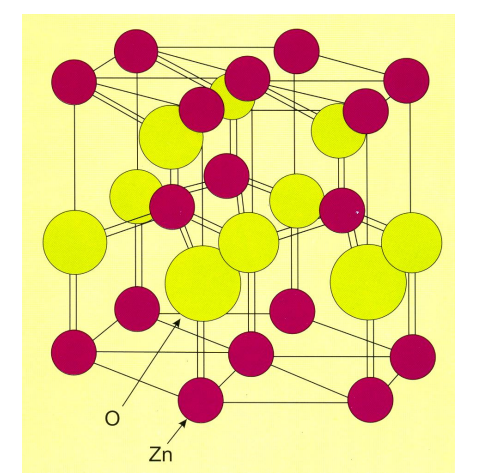

Figure 1. Wurtzite structure of $\mathrm{ZnO}$

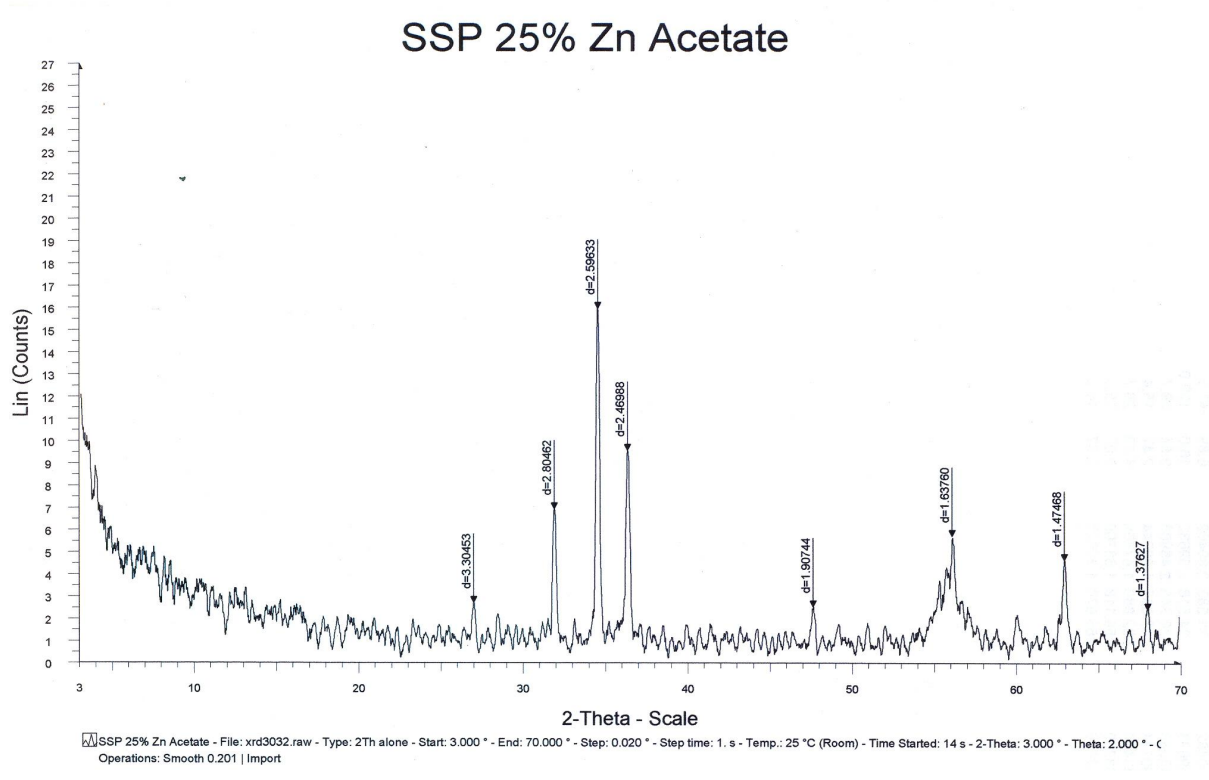

Figure 2. XRD pattern of $25 \%$ sol concentration nc-ZnO film on single side polished (SSP) Si wafer

SEM micrograph of these $10 \%, 15 \%$ and $25 \%$ zinc acetate concentration derived nc-ZnO thin films revealed the polycrystalline nature and smoothness of the deposited film with random spindle shaped, bunches of aligned nanorods as domains and clustering of these domains as nanowalls sol concentration increases as shown in Figure 3(a,b,c).

Proc. of SPIE Vol. 8549 85492D-2 


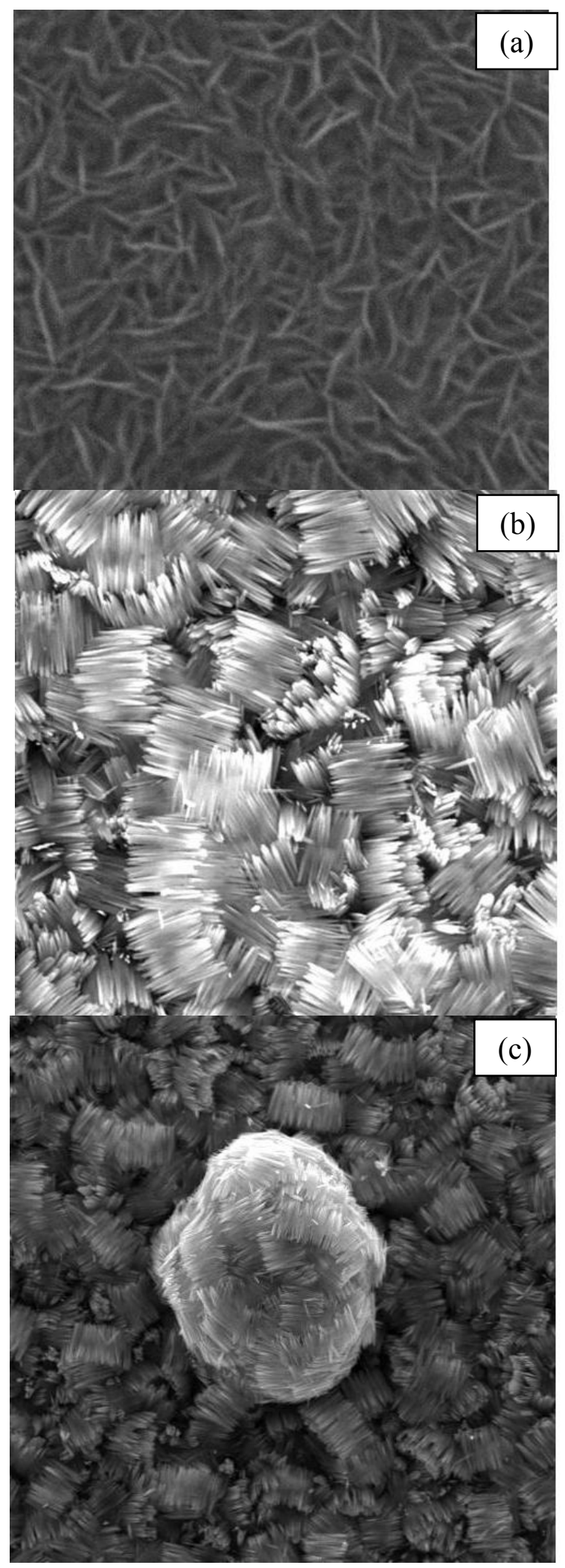

Figure 3. SEM images of (a) $10 \%$ (b) $15 \%$ and (c) $25 \% \mathrm{ZnO}$ nanorods

EDS spectrum showed strong peaks of $\mathrm{Zn}$ and $\mathrm{O}$ with weak peaks of $\mathrm{Si}$ substrate at the penetration depth of incident electron of $15 \mathrm{keV}$. The main lines due $\mathrm{ZnK} \alpha(8.64 \mathrm{keV})$ and $\mathrm{ZnK} \beta(9.57 \mathrm{keV})$ excitations and the peak for oxygen are observed and presented in Figure 4. 


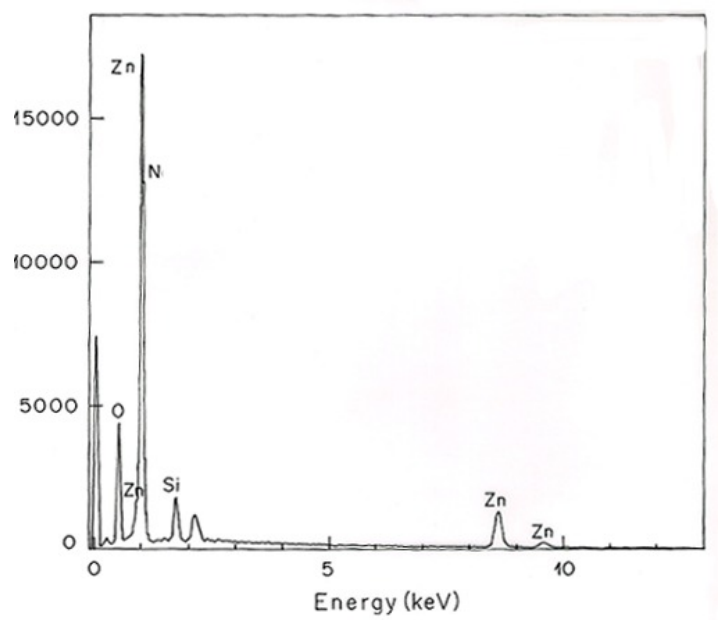

Figure 4. EDS of $25 \%$ zinc acetate sol concentration derived nc-ZnO thin film

Nanosized morphologies of $\mathrm{ZnO}$ are used to study the size-dependent PL phenomena [7]. Based on the basic theory of confinement effect, the confined ground-state excitonic energy (Eex ) versus the average particle size (R) can be expressed approximately by the following relation as:

$$
\text { Eex }=E g-\left(13.6 \mu / m_{e} \varepsilon_{r}^{2}\right)+\left(2 \pi^{2} \hbar^{2} /\left(m_{e}{ }^{*}+m_{h}{ }^{*}\right) R^{2}\right.
$$

Where $\mathrm{E}_{\mathrm{g}}$ is the band gap energy of bulk $\mathrm{ZnO}$, the second term is the binding energy ( $\left.60 \mathrm{meV}\right), \varepsilon_{\mathrm{r}}$ is the electricity constant and the third term is due to confinement of quantum structure/dot in which $\mathrm{m}_{\mathrm{e}}{ }^{*}$ and $\mathrm{m}_{\mathrm{h}}{ }^{*}$ are the reduced effective mass of electron and hole respectively. The observed blue shift in free exciton emission energy with change in morphology of these film films with sol concentration is attributed to the quantum confinement effect. This effect may arise from the difference of interactions involved, i.e. the photon-electron interaction in PL [8,9]

The excitation of $25 \%$ sol derived $\mathrm{ZnO}$ thin film with $241.01 \mathrm{~nm}$ radiation exhibits recombination of free excitons peak at $\sim 364$ $\mathrm{nm}$ as compared to bulk $\mathrm{ZnO}$ at $378 \mathrm{~nm}$ showing a blue shift of $14 \mathrm{~nm}$. This blueshift directly demonstrates that the assynthesized $\mathrm{ZnO}$ nanorods are already in the quantum confinement regime and this effect arises due to the suppression of defectrelated visible emission by the decrease in particles size to $\sim 10 \mathrm{~nm}$ or less and presence more atoms at the surface. The emission intensity decreases linearly with the change in morphology of the film.

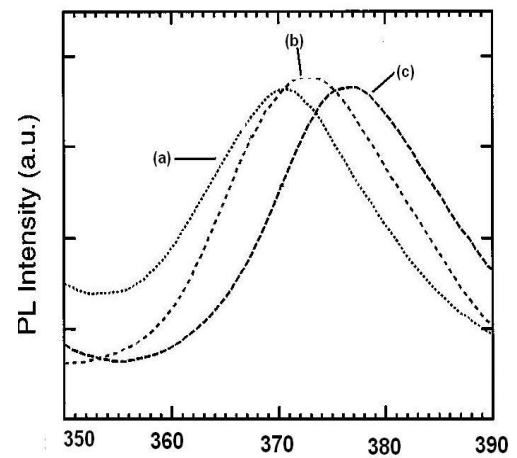

Figure5. PL Free exciton spectra of nc- $\mathrm{ZnO}$ thin (a) $10 \%$, (b) $15 \%$ and (c) $25 \%$ sol concentration

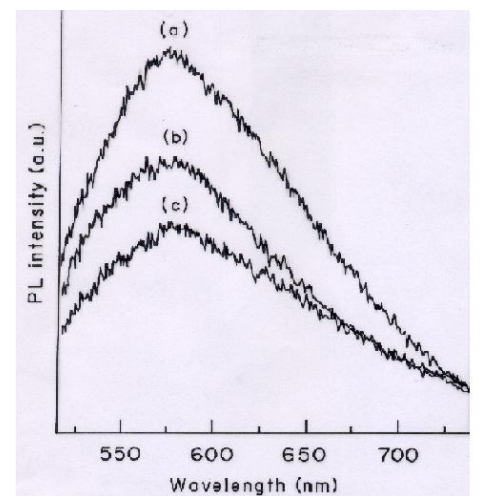

Figure6. Visible emission of nc-ZnO thin films (a)10\%, (b) $15 \%$ and $25 \%$ sol. concentration

The visible emission from these nc- $\mathrm{ZnO}$ thin films are observed $\sim 572 \mathrm{~nm}$ due to the recombination between oxygen vacancies, which act as deep level electron donor states, and the valence band as shown in Figure6. As we know that the effective mass for electrons in $\mathrm{ZnO}$ is much smaller than the effective mass for holes. So, the increase in energy of the conduction band is larger than the lowering of the valence band. This results in a significant shift in the energy of the deep level donor state in the quantum particles the energy of the donor state was independent of particle size and major the enlargement in the quantum particles appears over the valence band. 
UV-VIS spectrum of the $25 \%$ sol concentration grown nc-ZnO thin film recorded in $200-800 \mathrm{~nm}$ region shows exciton absorption peak is at $372 \mathrm{~nm}$ i.e. close to the expected value $378 \mathrm{~nm}$ of $\mathrm{ZnO}$.

\section{CONCLUSIONS}

In conclusion, $\mathrm{ZnO}$ nanorods were grown on the pre-oxidized silicon substrates by sol-gel process with different sol concentrations. XRD, SEM, EDS, PL and UV-VIS optical studies revealed the uniform, polycrystalline, wurtzite structure with preferential c-axis oriented growth and aligned along [002] plane. Obtained nanorods are in 20-30 nm in diameters. Nanowalls growth is observed in higher sol-concentration derived films due to clustering of nanorods in wall pattern. PL measurement showed excitonic transition from the conduction band to the valence band at $378 \mathrm{~nm}$. The blue shift due to quantum confinement effect is observed in the PL spectra of these films. This is induced by the phonon - electron interaction. The visible emission due to recombination of oxygen vacancies is also observed in the PL spectra ` $572 \mathrm{~nm}$. UV-VIS spectra showed excitons excitation at $373 \mathrm{~nm}$.

\section{ACKNOWLEDGEMENT}

The authors are grateful to Prof. R.C. Budhani, Director, NPL, New Delhi for his encouragement and permission to carry out this work.

\section{REFERENCES}

[1] Klingshirn C., "ZnO: Material, Physics and Applications”, Chem. Phys. Chem., 8, 782-803 (2007).

[2] Fonoberov V. A., Balandin A. A., "Origin of ultraviolet photoluminescence in $\mathrm{ZnO}$ quantum dots: Confined excitons versus surface-bound impurity exciton complexes" Appl. Phys. Lett.. 85, 5971-5973 (2004).

[3] Bouvy C., Marine W., Sporken R., Su B. L., "Photoluminescence properties and quantum size effect of ZnO nanoparticles confined inside a faujasite X zeolite matrix", Chemical Physics Letters 428, 312-316 (2006).

[4] Kiliani G., Schneider R., Litvinov D., Gerthsen D., Fonin M., R"udiger R., Leitenstorfer A., Bratschitsch R., "Ultraviolet photoluminescence of $\mathrm{ZnO}$ quantum dots sputtered at room-temperature", Optics Express, 19, 1641-1647 (2011).

[5] Gür E., Tüzemen S., Meral K., Onganer Y., “ Oxygen deficiency effects on recombination lifetime and photoluminescence characteristics of zno thin films; correlation with crystal structure", Applied Physics A: Materials Science \& Processing, 94, $549-554$ (2009).

[6] Wong K. M., Fang Y., Devaux A., Wen L., Huang J., Cola L. D., Lei Y., "Assorted analytical and spectroscopic techniques for the optimization of the defect-related properties in size-controlled ZnO nanowires", Nanoscale, 3, 4830-4839 (2011).

[7 ] Zhao J., Han Z., Lu L., Wang X., Chen J., "Synthesis and photoluminescence properties of ZnO powder by solution combustion method", J. Mater Sci: Mater Electron, 22, 1361-1365 (2011).

[8] Fu Z.D., Cui Y.S., Zhang S.Y., Chen J., Yu D.P., Zhang S.L., Niu L. and Jian J.Z., "Study on the quantum confinement effect on ultraviolet photoluminescence of crystalline $\mathrm{ZnO}$ nanoparticles with nearly uniform size" Appl. Phys. Lett. 90, 263113 (2007).

[9] Cao B., Cai W., Zeng H., "Temperature-dependent shifts of three emission bands for ZnO nanoneedle arrays" Appl. Phys. Lett. 88, 161101 (2006). 